\title{
The outflow activity of the protostars in S140 IRS ${ }^{\star}$
}

\author{
T. Preibisch ${ }^{1}$ and M. D. Smith ${ }^{2}$ \\ 1 Max-Planck-Institut für Radioastronomie, Auf dem Hügel 69, 53121 Bonn, Germany \\ 2 Armagh Observatory, College Hill, Armagh BT61 9DG, Northern Ireland
}

Received 24 October 2001 / Accepted 30 November 2001

\begin{abstract}
The S140/L1204 cloud contains a deeply embedded region of star formation and a powerful molecular outflow. In this paper, we present images of the S140 region obtained in the light of the $2.12 \mu \mathrm{m}$ molecular hydrogen emission line and adjacent continuum. Our images reveal several knots of $\mathrm{H}_{2}$ line emission originating from shocked material close to IRS 1 as well as further out. Strong $\mathrm{H}_{2}$ shock emission is found north-east of IRS1 (at position angle of $\sim 20^{\circ}-30^{\circ}$ ), as well as to the south-west of IRS1 (at position angles around $\sim 190^{\circ}-$ $220^{\circ}$ ), clearly demonstrating the presence of outflow activity in the north-east/south-west direction. We also find patches of $\mathrm{H}_{2}$ emission several arcminutes away from IRS1 at a position angles of $\sim 150^{\circ}$ and $340^{\circ}$, i.e. in directions consistent with the previously known north-west/south-east molecular outflow. Our results therefore provide evidence for the existence of two distinct bipolar outflow systems originating simultaneously from IRS 1. We also discuss general aspects of the star formation process in the S140 region. An inferred high ratio of stellar to gas mass suggests that the outflows have dispersed most of the cloud mass.
\end{abstract}

Key words. stars: individual: S140 IRS 1 - stars: individual: S140 IRS 3 - stars: formation - stars: winds, outflows

\section{Introduction}

S140 is an HII region at the southeast edge of the L1204 dark cloud at a distance of $\sim 900$ pc (cf. Crampton \& Fisher 1974). It is part of a cloud complex located at the edge of a prominent infrared emission ring, known as the Cepheus ring. This ring is probably the result of supernova explosions and stellar winds from massive stars in the open cluster NGC 7160 at the center of the ring (cf. Kun et al. 1987; de Zeeuw et al. 1999).

The S140 cloud contains a small cluster of highly obscured, optically invisible infrared sources, originally detected by Rouan et al. (1977). The $20 \mu \mathrm{m}$ observations by Beichman et al. (1979) revealed three individual infrared sources in this region. The brightest source is called IRS 1, and the two other sources IRS 2 and IRS 3 are located $\sim 17^{\prime \prime}$ north and $\sim 9^{\prime \prime}$ east of IRS 1 . The infrared spectra of these sources are rising steeply between $10 \mu \mathrm{m}$ and $50 \mu \mathrm{m}$ (Lester et al. 1986), demonstrating that they are deeply embedded young stellar objects associated with circumstellar material. The luminosities of IRS 1, 2, and 3 were estimated at 5,3 , and $2 \times 10^{3} L_{\odot}$ respectively

Send offprint requests to: T. Preibisch, e-mail: preib@mpifr-bonn.mpg.de

* Based on observations obtained at the German-Spanish Astronomical Centre, Calar Alto, operated by the Max-PlanckInstiute for Astronomy, Heidelberg, jointly with the Spanish National Commission for Astronomy.
(Lester et al. 1986), suggesting stellar masses between $\approx 6 M_{\odot}$ and $\approx 10 M_{\odot}$.

S140 IRS is the source of a strong molecular outflow. A bipolar CO outflow was first detected by Blair et al. (1978) and later studied in more detail, e.g. by Hayashi \& Murata (1992), Minchin et al. (1993), and Wilner \& Welch (1994). S140 IRS 1 lies just in the middle between the blue- and red-shifted outflow lobes which have position angles of $160^{\circ}$ and $340^{\circ}$, respectively. While the morphology of the molecular line emission in all these observations is consistent with the idea that IRS 1 is the driving source of the outflows, the spatial resolution of the maps is far too coarse to exclude the possibility that IRS 2 or IRS 3 also contribute to the outflow activity.

First high-resolution infrared observations of IRS 1 were obtained by Hoare et al. (1996) and revealed diffuse emission extending to the south-east of the source. In recent high-resolution studies of the S140 star forming region we have presented bispectrum speckle interferometric $K$-band images of IRS 1 (Schertl et al. 2000; Weigelt et al. 2002). We found a bright elongated clumpy structure pointing away from IRS 1 in a direction $\left(160^{\circ}\right)$ very consistent with that of the blue-shifted $\mathrm{CO}$ outflow lobe. We interpreted this feature as the clumpy inner edge of a cavity in the circumstellar envelope around IRS 1 , which has been excavated by the south-eastern wing of the strong $160^{\circ} / 340^{\circ}$ molecular outflow. We also found three arc-like structures to the north-east of IRS 1 at position 
angles of $\sim 15^{\circ}-25^{\circ}$ and suggested that these structures represent cavities created by material flowing away from IRS 1 in north-eastern directions. These high-resolution data revealed unique insights into the detailed morphology of the circumstellar structures around IRS 1. However, as they had to be taken in a broad-band $K^{\prime}$-filter, they did not allow us to discriminate between continuum emission (e.g. scattered stellar light from IRS 1 and thermal dust emission from the inner circumstellar matter) on the one hand and line emission from shocked gas on the other hand. Therefore, the interpretation of the observed structures was not conclusive.

Outflows and jets from young stellar objects strongly interact with the surrounding material. Shocks are generated where the flow, ejected from the protostar, impacts with the dense environment. The $2.12 \mu \mathrm{m} \nu=1-0 \mathrm{~S}(1)$ ro-vibrational emission line of molecular hydrogen is a convenient tracer of these shocks (e.g. Smith 1993; Eislöffel et al. 2000). Images taken in the light of the molecular hydrogen emission line can therefore reveal the structure and morphology of the outflow, and provide important constraints on the nature of the flow collimation and variability. Motivated by these ideas, we have imaged the S140 IRS region in the molecular hydrogen line emission and adjacent continuum. In Preibisch et al. (2001) we presented a study combining high-resolution speckle images and molecular hydrogen line emission images of S140 IRS 3. In this paper we present our $\mathrm{H}_{2}$ results on IRS 1 and consider general aspects on the star formation process in the S140 IRS region.

\section{Observations}

The infrared data presented here were obtained in October 2000 with the Omega Prime wide-field near-IR camera (Bizenberger et al. 1998) on the Calar Alto $3.5 \mathrm{~m}$ telescope. The camera uses a $1024 \times 1024$ pixel $\mathrm{HgCdTe}$ array. At $0.4^{\prime \prime}$ per pixel, the field-of-view is $6.7^{\prime} \times 6.7^{\prime}$. Images were taken through the NB2122 filter, a $1 \%$ filter centred on the $v=1-0 \mathrm{~S}(1)$ line of the $\mathrm{H}_{2}$ molecule at $2.12 \mu \mathrm{m}$, and through a broad-band $K^{\prime}$ filter $(1.944-2.292 \mu \mathrm{m})$. We took images at 6 different dither positions with positional shifts of $40^{\prime \prime}$; at each position a series of 10 images with $30 \mathrm{~s}$ (1.677 s for the $K^{\prime}$ filter) exposure time was taken. We also obtained images through a standard $J$-band filter.

The weather conditions during our observations were good, the seeing (as measured by the FWHM of the PSF in our images) was $\sim 1.2^{\prime \prime}-1.6^{\prime \prime}$. Standard data reduction techniques were used to sky subtract, flat field, and mosaic the data (cf. McCaughrean et al. 1994). Then, the NB2122 filter image was carefully aligned with the $K^{\prime}$ filter image via several point sources in the outer parts of the image. Finally, we subtracted the $K^{\prime}$ filter image from the NB2122 filter image in order to create the $\mathrm{H}_{2}$ emission line image. The field-of-view of our final mosaic image is $8^{\prime} \times 8^{\prime}$.

\section{3. $\mathrm{H}_{2}$ emission in and around S140 IRS}

The $\mathrm{H}_{2}$ emission related to S140 IRS 3 has already been investigated in a previous paper (Preibisch et al. 2001); here we will therefore focus on the $\mathrm{H}_{2}$ emission related to IRS 1.

\subsection{Inner part}

Our image reveals many patches of $\mathrm{H}_{2}$ line emission in and around the S140 IRS region. The strongest $\mathrm{H}_{2}$ emission knots are found within $15^{\prime \prime}$ of IRS 1 . Figure 1 shows the central part of our $K^{\prime}$-band image of S140 IRS onto which the contours of the continuum-subtracted $2.12 \mu \mathrm{m} \mathrm{H}_{2}$ line emission are overlaid. The most prominent knots of $\mathrm{H}_{2}$ line emission are marked with numbers $\mathbf{1}$ to $\mathbf{8}$.

\subsection{1. $\mathrm{H}_{2}$ emission north-east of IRS 1}

Knot $\mathbf{1}$ is an elongated feature of strong $\mathrm{H}_{2}$ emission north-east of IRS 1 . The emission peak is found $13^{\prime \prime}$ away from IRS 1 at a position angle of $\sim 28^{\circ}$. The $\mathrm{H}_{2}$ emission is strongly elongated in the north-south direction and very well aligned with the eastern edge of the arc-structure north-east of IRS 1 in the $K^{\prime}$ band image. It is important to note that the peak of the $\mathrm{H}_{2}$ emission does not coincide with the brightest features seen in the $K^{\prime}$ band continuum, but is offset by about $3^{\prime \prime}$. The weaker extensions of the $\mathrm{H}_{2}$ emission cover the full extent of the arc structure. Another weak peak of $\mathrm{H}_{2}$ emission (knot 2) is found $6^{\prime \prime}$ north-east of IRS 1 and is well aligned with the southern part of the arc-structure.

\subsection{2. $\mathrm{H}_{2}$ emission south of IRS 1}

The area south-west of IRS 1 contains a ridge of strong $\mathrm{H}_{2}$ emission, roughly oriented in the east-west direction. The two strongest maxima are knot 4 , located $6^{\prime \prime}$ south of IRS 1 at a position angle of $\sim 185^{\circ}$, and knot $\mathbf{5}$, located $8^{\prime \prime}$ south-west of IRS 1 at a position angle of $225^{\circ}$. These $\mathrm{H}_{2}$ knots coincide very well with the brightest parts of the $K^{\prime}$ band continuum nebulosity. The detection of these $\mathrm{H}_{2}$ emission knots provides evidence for material flowing from IRS 1 to the south-western direction. This can be quite naturally explained as the counterpart of the $20^{\circ}$ outflow.

Another patch of $\mathrm{H}_{2}$ emission ( $k$ not 3 ) is seen $8^{\prime \prime}$ southeast of IRS 1 at position angles in the range $\sim 135-160^{\circ}$. This feature is probably related to the well known $160^{\circ}$ molecular outflow.

\subsection{3. $\mathrm{H}_{2}$ emission east and north-east of IRS 3}

This area contains the two $\mathrm{H}_{2}$ emission knots 6 and 7 . A detailed study of the continuum and the $\mathrm{H}_{2}$ line emission in the vicinity of IRS 3 has already been presented in Preibisch et al. (2001). 

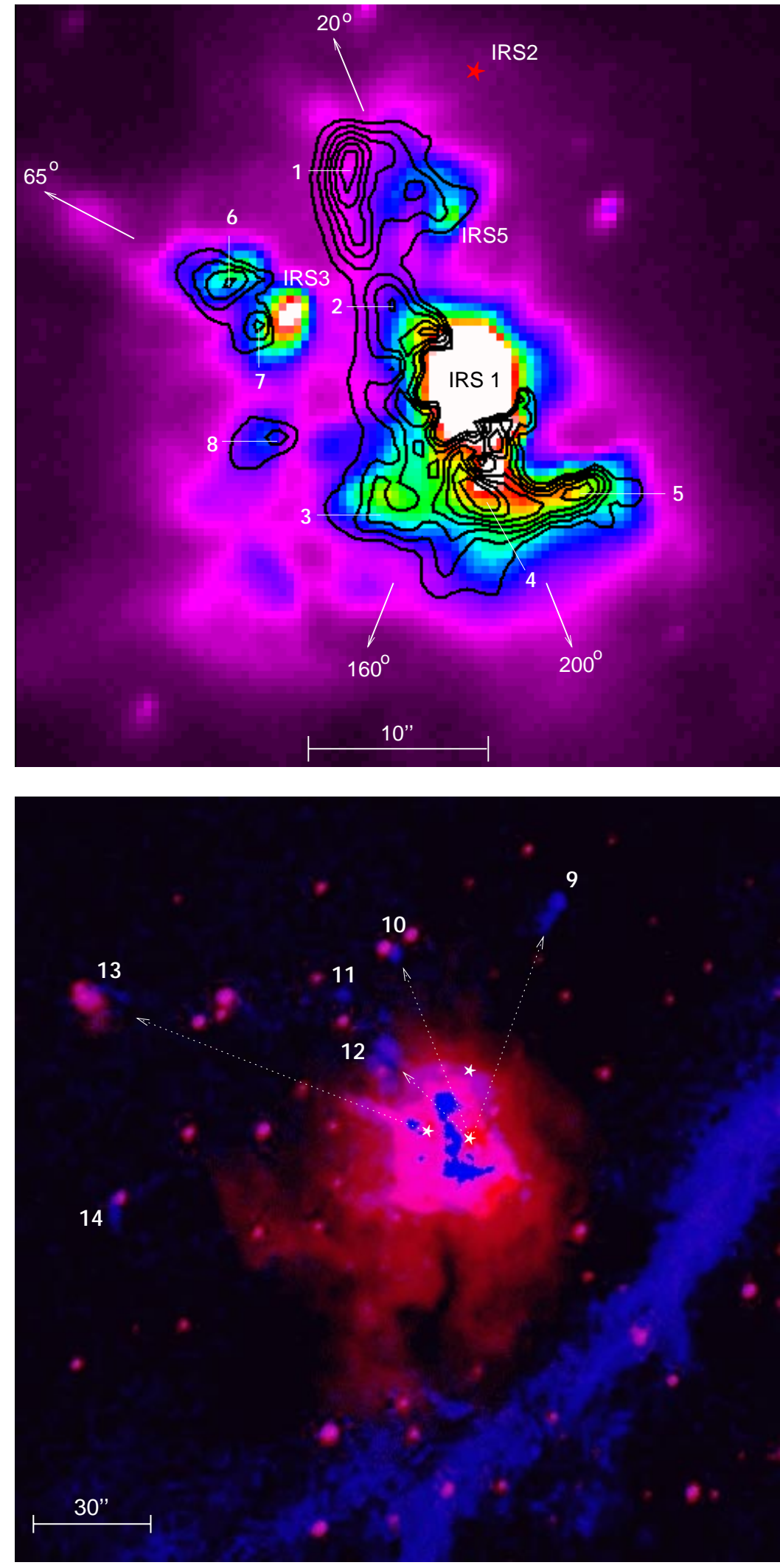

Fig. 1. The color image shows our $K^{\prime}$-band image of the S140 IRS region with a rainbow color-table; the central point source IRS 1 is saturated. The black contour lines show the continuum-subtracted $2.12 \mu \mathrm{m} \mathrm{H}_{2}$ line emission image. Contour levels are 10, 15, 20,2 5, 30, 35, and $40 \sigma$ above the median in the $\mathrm{H}_{2}$ image. In the immediate vicinity of IRS 1 , our $\mathrm{H}_{2}$ emission map is slightly affected by small artifacts due to imperfect continuum subtraction. We therefore will only discuss features more than $4^{\prime \prime}$ away from IRS 1 . The eight most prominent $\mathrm{H}_{2}$ knots are marked with numbers $\mathbf{1}$ to $\mathbf{8}$. The position of the deeply embedded infrared source IRS 2, which is invisible in the near-infrared, is marked by the red asterisk. (This figure is available in color in the online version.)

Fig. 2. Color-composit image of a $3.2^{\prime} \times 3.2^{\prime}$ area around S140 IRS. The red color plane represents the $K^{\prime}$ band image, the blue color plane the continuum-subtracted $2.12 \mu \mathrm{m} \mathrm{H}_{2}$ line emission image. The most prominent patches of $\mathrm{H}_{2}$ emission around S140 IRS are marked. The three white asterisks mark the positions of IRS 1 , IRS 2, and IRS 3. (This figure is available in color in the online version.)

\subsection{Outer environment}

Figure 2 shows a larger field around S140 IRS. The most prominent structure in the $\mathrm{H}_{2}$ line emission image is the bright rim of the the S140 HII region, crossing the south-western edge of our image. This HII region is powered by the B0 star HD 211880, located $\sim 8^{\prime}$ south-west of $\mathrm{S} 140$ IRS. The $\mathrm{H}_{2}$ line emission results from fluorescent excitation (cf. Tanaka et al. 1989). 
In the northern and eastern part of Fig. 1 we find several patches of $\mathrm{H}_{2}$ line emission at distances between $15^{\prime \prime}$ and $90^{\prime \prime}$ away from IRS 1 . We have assigned numbers $\mathbf{9}$ to 14 to those $\mathrm{H}_{2}$ emission knots in which the peak surface brightness exceeds the noise level in the continuum subtracted image by more than $5 \sigma$.

Knot 9 is located $70^{\prime \prime}$ north-west of IRS 1 at a position angle of $340^{\circ}$. It is clearly elongated in a direction pointing towards IRS 1 . Therefore, we conclude that this $\mathrm{H}_{2}$ emission knot is related to the well known $340^{\circ} \mathrm{CO}$ outflow from IRS 1 . Knot 9 is the first infrared evidence for the receding lobe of the $160^{\circ} / 340^{\circ}$ outflow.

Knot $\mathbf{1 0}$ is found $53^{\prime \prime}$ north-east of IRS 1 at a position angle of $20^{\circ}$. This direction is in very good agreement with the orientation of the arc complex north-east of IRS1 described in the speckle images by Weigelt et al. (2002).

Knots $11\left(50^{\prime \prime}\right.$ north-east of IRS 1 at a position angle of $\left.40^{\circ}\right)$ and $12\left(30^{\prime \prime}\right.$ north-east of IRS 1 at a position angle of $45^{\circ}$ ) are probably also related to the north-eastern outflow.

Knot 13 is related to the bow-shock like nebulosity $90^{\prime \prime}$ north-east of IRS 3 . Its position angle measured from IRS 3 is $65^{\circ}$, exactly the direction of the precessing outflow from IRS 3 detected by Preibisch et al. (2001).

Knot 14 is located $90^{\prime \prime}$ south-east of IRS 1 at a position angle of $100^{\circ}$. It is not clear whether this patch is related to IRS 1 or IRS 3.

\section{The outflow activity of S140 IRS 1}

The combination of our $\mathrm{H}_{2}$ data with previous observational results, allows us to draw a comprehensive picture of the outflow activity of S140 IRS 1 on a wide range of spatial and temporal scales:

- Our high-resolution speckle interferometric observations provide information about the detailed morphology of the circumstellar material in the immediate vicinity of IRS 1, on spatial scales from $\sim 100 \mathrm{AU}$ to $\sim 10000 \mathrm{AU}$.

- The high-resolution radio continuum observations provide information about the ionized stellar winds and jets close to the central source.

- The $\mathrm{H}_{2}$ line emission images show those regions where the outflowing material from the protostars impacts with the dense environment. Because of the relatively short radiative cooling time (on the order of a few years) of the $\mathrm{H}_{2}$ $\nu=1-0 \mathrm{~S}(1)$ transition, the molecular hydrogen emission illustrates the current shock-activity in the outflows.

- The radio molecular line emission observations provide information about the large scale structure of the outflows on spatial scales of $\sim 50000 \mathrm{AU}$ and the long-term effects of the outflows on the ambient material

\subsection{The $160^{\circ} / 340^{\circ}$ outflow}

The presence of the large scale $160^{\circ} / \mathbf{3 4 0}^{\circ}$ outflow was already well established from radio line observations. In the radio maps, outflowing gas can be seen at least up to distances of $1^{\prime}$ (54000 AU) from IRS 1 (e.g.
Minchin et al. 1993). Our high-resolution speckle observations (Schertl et al. 2000; Weigelt et al. 2002) revealed the detailed structure of the cavity through which the material in the $160^{\circ}$ outflow lobe is flowing on scales of $90-1800 \mathrm{AU}$. Our molecular hydrogen emission line images show only two relatively weak $\mathrm{H}_{2}$ knots associated with this outflow, knots 3 and $\mathbf{9}$. This suggests that there are currently not many strong shocks in this outflow, perhaps because the path of the outflow is already well cleared and the material is flowing away without producing strong shocks. However, the detection of $\mathrm{H}_{2}$ shock emission in this outflow is aggravated due to the inclination of the flow and projection effects: In the south-eastern part, where the outflow is directed towards us, $\mathrm{H}_{2}$ emission very close to $\left(\$ 2^{\prime \prime}\right.$ away from) IRS 1 would be projected onto the very bright emission of the cavity. In the north-western part of this outflow, $\mathrm{H}_{2}$ shock emission might be present, but hidden behind the dense circumstellar material around IRS 1 . The fact that the high resolution radio continuum observations presented by Schwartz (1989) and Hoare \& Muxlow (1996) do not show any sign of the $160^{\circ} / 340^{\circ}$ outflow, supports our assumption that this outflow is probably currently not very active.

A rough estimate of the kinematic age of this outflow can also be made: from the projected distance of knot $\mathbf{9}$, an assumed velocity of $\sim 100 \mathrm{~km} \mathrm{~s}^{-1}$, and assuming an inclination angle of $i \gtrsim 45^{\circ}$, we find a lower limit of $\tau \gtrsim$ 6000 years.

\subsection{The $20^{\circ} / 200^{\circ}$ outflow}

The existence of a $\mathbf{2 0}^{\circ} / \mathbf{2 0 0}^{\circ}$ outflow has not so far been well established. Radio line observations had shown a relatively weak low-velocity wing in the $\mathrm{CO}$ and $\mathrm{HCO}^{+}$ emission that indicated outflowing material in the $20^{\circ}$ direction, but no reliable evidence for an outflow in the $200^{\circ}$ direction. The high-resolution radio continuum maps of S140 IRS 1 presented by Schwartz (1989) revealed a strongly elongated jet-like appendage extending from IRS 1 in the south-west direction (position angle $225^{\circ}$ ), and a diffuse radio source (VLA 4) south-west of IRS 1 (position angle $217^{\circ}$ ) which may be a radio Herbig-Haro object. These features were interpreted as evidence for ejection of material from IRS 1 in the south-western direction. The high-resolution radio observations by Hoare \& Muxlow (1996) showed a strongly elongated structure oriented in the $44^{\circ} / 224^{\circ}$ direction. It was interpreted as a highly collimated jet, probably being driven by a disk wind. The $8.4 \mathrm{GHz}$ VLA observations of S140 presented by Tofani et al. (1995) revealed an interesting S-shape for the emission within $1^{\prime \prime}$ of IRS 1 . While the core of the emission is oriented in the $44^{\circ} / 224^{\circ}$ direction, the position angle of the north-eastern tip of the $8.4 \mathrm{GHz}$ emission is $20^{\circ}$, in very good agreement with the position angle of the outflow cavities we found in the speckle images.

Our high-resolution speckle observations (Weigelt et al. 2002) revealed three arc-like structures with 
position angles of $10^{\circ}, 20^{\circ}$, and $25^{\circ}$ north-east of IRS 1 . The shapes of these arcs could be well reproduced by a semi-analytical model of jet-driven flows, in which prompt entrainment occurs at the head of the traveling bow shock. This strongly suggested a flow of material in this direction, but could not be considered as a clear proof. The result that the eastern edge of the supposed cavities is actually associated with strong $\mathrm{H}_{2}$ emission, confirms our previous interpretation that these cavities have been created by outflowing material from IRS 1 in the $\sim 20^{\circ}$ direction.

Our $\mathrm{H}_{2}$ line emission image reveals numerous knots along the paths of the $\mathbf{2 0}^{\circ} / \mathbf{2 0 0}^{\circ}$ outflow: knots $\mathbf{1}, \mathbf{2}, \mathbf{1 0}$, $\mathbf{1 1}$, and $\mathbf{1 2}$ are related to the $20^{\circ}$ flow, knots $\mathbf{4}$ and $\mathbf{5}$ to the $200^{\circ}$ flow. These knots span a range of position angles from $20^{\circ}$ to $45^{\circ}$, which is consistent with the orientation of the elongated radio emission immediately around IRS 1.

The numerous $\mathrm{H}_{2}$ shocks in these directions indicate that the outflow currently interacts very strongly with its surroundings. Perhaps the outflow is currently in the process of clearing its way through the ambient material. The rather wide range of position angles of the individual $\mathrm{H}_{2}$ knots north-east of IRS 1 suggest a pulsed nature for the outflow and directional variability. A wiggling jet in the NE/SW direction is also suggested by the radio continuum and maser emission on the scale of $0.5^{\prime \prime}$ (Tofani et al. 1995), which extends in the $45^{\circ}$ direction i.e. towards $\mathrm{H}_{2}$ knot 2 . The temporal variability of the outflow direction may be caused by the precession of the circumstellar disk around the outflow source which is a member of a non-coplanar binary system (see Weigelt et al. 2002 for details; for the theoretical background of this model see e.g. Papaloizou \& Terquem 1995; Terquem 1998; Bate et al. 2000).

A rough estimate of the kinematic age of this outflow, based on the distance of knot 10, an assumed inclination angle of $i \sim 0^{\circ}$ and a velocity $\sim 100 \mathrm{~km} \mathrm{~s}^{-1}$, yields $\tau \sim$ 2300 years for the $\mathbf{2 0}^{\circ} / \mathbf{2 0 0}^{\circ}$ outflow. This is nearly three times younger than the lower limit to the kinematic age of the $160^{\circ} / \mathbf{3 4 0}^{\circ}$ outflow.

\section{General considerations on the star formation process in the S140 IRS region}

\subsection{The stellar population of S140 IRS}

Near-infrared images (e.g. Fig. 2; see also Hodapp 1994) show a small cluster of fainter point sources around the nebulosity surrounding IRS 1 . It seems very likely that most of these objects are associated with the S140 star forming region for two reasons: first, the extinction of the S140 molecular cloud was estimated to be up to $A_{V} \sim 100$ mag based on the determination of $N\left(\mathrm{C}^{18} \mathrm{O}\right)$ by Minchin et al. (1995); it therefore provides an essentially opaque shield for the stellar background, and we can assume that all stars we see in this region are either foreground stars or associated with the S140 cloud. Second, the optical images of the region show only very few stars within a few arcminutes of the position of IRS 1, clearly

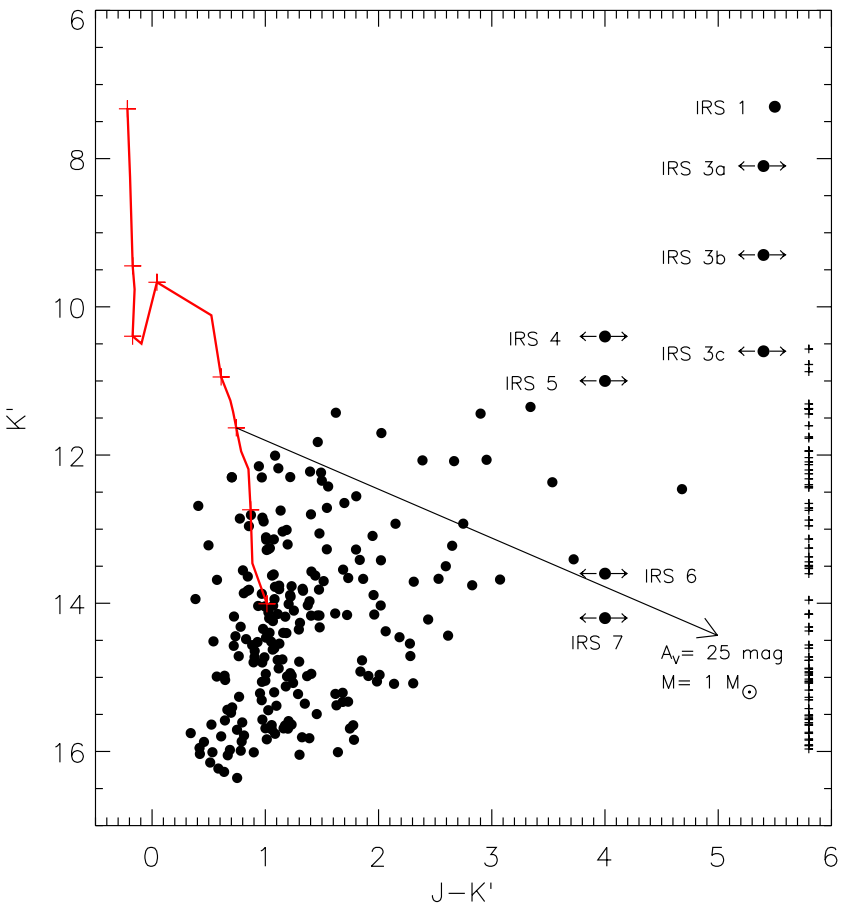

Fig. 3. Color-magnitude diagram of stars in the S140 IRS region (solid dots). We have included the individual objects resolved in our speckle images; for these, no colors can be determined and we assume each component to have the same color as reported for the unresolved systems. All objects for which only $K^{\prime}$-band magnitudes could be derived are plotted as small crosses at $J-K^{\prime}=5.8$. The thick solid line in the left part of the diagram shows theoretical colors and magnitudes based on the PMS models of Palla \& Stahler (2000) and Bernasconi \& Maeder (1996) for an age of $1 \times 10^{6} \mathrm{yr}$ for masses from $0.1 M_{\odot}$ to $15 M_{\odot}$. Tick marks are shown for masses of $0.1,0.4,1,2$, $3,4,6$, and $15 M_{\odot}$. The long arrow shows the reddening path for $A_{V}=25$ mag starting at the location for a $1 \times 10^{6} \mathrm{yr}$ old $1 M_{\odot}$ star.

demonstrating that the foreground population cannot be very large.

Our deep $J$ - and $K^{\prime}$-band data offer a good opportunity to examine the stellar population of the S140 starforming region. For this, we used DAOPHOT to locate point sources in our deep $J$ - and $K^{\prime}$-band images and performed aperture photometry. The central $20^{\prime \prime}$ region had to be excluded from this analysis because of the extremely strong diffuse emission in the image center. In the circular region with radius $4^{\prime}$ around IRS 1 , photometric data could be determined for 388 stars in the $K^{\prime}$-band image and for 272 stars in the $J$-band image. The magnitudes were calibrated via observations of other regions and comparison with the corresponding magnitudes from the 2MASS catalogue. Due to the strong diffuse emission in the S140 IRS region the accuracy of the photometry is limited to $\sim 0.2 \mathrm{mag}$. Nevertheless, this is sufficiently accurate to consider the positions of the stars in the colormagnitude diagram.

Our color-magnitude diagram (CMD) is shown in Fig. 3. The comparison with the expected colors and 
magnitudes for pre-main sequence stars shows that our sample of $K^{\prime}$-band sources should be complete for $1 M_{\odot}$ stars suffering up to 25 mag of visual extinction (expected magnitude $K^{\prime} \sim 14.4$ ). The number of stars for which the NIR data are consistent with being cluster members with $M \geq 1 M_{\odot}$ is 39 (this includes 20 stars with $K^{\prime}<12.5$ for which we could only measure $K^{\prime}$-band magnitudes.) Four out of these 39 stars, namely IRS 1, IRS 2, IRS 3a, and IRS $3 \mathrm{~b}$, are probably more massive than $5 M_{\odot}$.

We can now compare these numbers with the expectation from the field star IMF. In the IMF representation by Scalo (1998), which has the form

$$
\frac{\mathrm{d} N}{\mathrm{~d} M} \propto\left\{\begin{array}{rrrr}
M^{-1.2} & \text { for } & 0.08 & <M / M_{\odot}<1 \\
M^{-2.7} & \text { for } & 1 & <M / M_{\odot}<10
\end{array}\right.
$$

the presence of 4 stars in the $[5 \ldots 10] M_{\odot}$ range would imply a total number of 83 stars with masses between $1 M_{\odot}$ and $10 M_{\odot}$. The IMF representation given by Kroupa (2001) which has the form

$\frac{\mathrm{d} N}{\mathrm{~d} M} \propto \begin{cases}M^{-1.3} & \text { for } \quad 0.08<M / M_{\odot}<0.5 \\ M^{-2.3} & \text { for } \quad 0.5<M / M_{\odot}<10\end{cases}$

would predict a total number of 48 stars in the $[1-10] M_{\odot}$ range. Our detection of 39 stars above $1 M_{\odot}$ is lower than both predictions. However, we have to take into account that we certainly miss some fraction of low-mass members, for two main reasons: first, our photometry does not cover the central $20^{\prime \prime}$ area due to extremely strong diffuse emission. Second, our sample can be expected to be complete only up to $A_{V} \lesssim 25 \mathrm{mag}$, while the total cloud extinction near the center is probably $A_{V} \approx 100$ mag. This suggests that our images probably reveal only about half of the low mass members in the cloud. Considering this, we conclude that the number of stars we see in our image is not inconsistent with the assumption that the cluster mass function follows the field star IMF.

\subsection{Stellar masses versus cloud mass}

We can estimate the total mass of the stars in the cluster by assuming that the mass function is the same as the field star IMF (as demonstrated above). In that case, the total mass of all stars $\left(0.1-10 M_{\odot}\right)$ in the cloud would be $\sim 340 M_{\odot}$ based on the Scalo (1998) IMF and $\sim 240 M_{\odot}$ based on the Kroupa (2001) IMF.

We can estimate the total mass of the stars in the cluster in the following way: For a very conservative lower limit, we consider only the objects IRS 1-IRS 7. The sum of the estimated masses of these objects is $\sim 40 M_{\odot}$.

The mass of the gas and dust in the S140 IRS cloud has been determined in various radio and submm studies. For example, Hayashi \& Murata (1992) derived a mass of $60 M_{\odot}$ within a radius of $r=0.15 \mathrm{pc}\left(\widehat{=} 34^{\prime \prime}\right)$. Van der Tak et al. (2000) found $M=46 M_{\odot}$ in $r=0.15 \mathrm{pc}$ from submm maps. Bally \& Lada (1983) derived a total mass of $\sim 64 M_{\odot}$ within $r=0.46$ pc $\left(\widehat{=} 1.7^{\prime}\right)$ from CO observations. From these numbers one can see that the mass of the stars is considerably higher than the cloud mass.
A similar result is obtained for the center of the starforming region: Considering only the objects IRS 1-IRS 7, the sum of their estimated masses is $\sim 40 M_{\odot}$. On the other hand, the mass of the three submm cores within $25^{\prime \prime}$ of IRS 1 is only $\sim 29 M_{\odot}$ (Minchin et al. 1995).

These considerations make it quite clear that the inferred total mass in young stars exceeds the mass in gas significantly. This implies that a high fraction of the original cloud mass has been converted to stars or that the formation of a protostar leads to the escape of a much larger mass.

\subsection{Cloud destruction by outflows?}

In S 140, there is clear evidence for the ejection of a large fraction of the cloud. Numerous outflows are found here in the warm shocked molecular gas, which reveals that even the present outflows contain sufficient thrust to eject material from the cloud. This is not surprising given that $10-30 \%$ of the mass accreting from the dense envelope around a core must be ejected into the jets observed from Class 0 protostars (e.g. Smith 2000) and the jet speed is of order $100 \mathrm{~km} \mathrm{~s}^{-1}$. The jets' thrust is thus sufficient to gravitationally unbind roughly ten times more mass than goes into the protostar. Hence, the total mass of the initial cloud out of which the stars formed may have been much larger than the sum of the star + gas mass presently found.

Given this picture, outflow activity must have influenced the cloud for its full lifetime. We could expect perhaps 100 outflows within a few million years. This would still be detectable in the CO rotational lines which contain a long-term record of outflow activity. And, indeed, the CO high-velocity gas possesses a complex distribution, consisting of numerous widely spread clumps, but bordered in the south and west by high density gas.

A problem with jet/outflow feedback scenarios is that a well collimated outflow only influences its immediate surroundings and not the cloud as a whole. It is not plausible to assert that an outflow can provide turbulent support to a cloud since turbulence decays faster than it could spread laterally, from an outflow, across a cloud given the rapid decay of turbulence found in MHD simulations (e.g. Mac Low 1999). Hence, even though the momentum inherent in the outflows may be sufficient (Yu et al. 2000), they will not be present to support the cloud as it evolves. A large number of outflows, however, distributed over space and the cloud life, may still be effective in dispersing the cloud with the following argument. If each outflow is represented as a cone with a half opening angle of just $10^{\circ}$, then 103 outflows would fill the cloud volume. Hence, over the cloud lifetime, and given random outflow directions over the cloud lifetime, a large fraction of the cloud can be ejected.

In contrast, the $\mathrm{H}_{2}$ gas is observed from hot shocks which are driven by the latest energetic outflows. Even so, we have found here evidence for several outflow directions, related to at least three outflows. The kinematic ages of 
the outflow have been estimated to be just a few thousand years. We suspect that the outflows are much older and penetrate far into the surrounding lower density halo where they are not detectable because of both the low density and possibly a low molecular fraction. Therefore, deep optical $\mathrm{H} \alpha$ imaging in the $\mathrm{NE}$ region beyond $4^{\prime}$ from IRS1 could reveal Herbig-Haro objects.

\subsection{Triggered star formation}

Triggered star formation by external compression ${ }^{1}$ might also lead to an enhancement of the star formation efficiency. This could also contribute to the high ratio of stellar mass versus gas mass we found above for the S140 region.

The S140 region is probably a good example for triggered star formation. A sequence of triggered star formation events in the Cepheus bubble has been suggested by Patel et al. (1998): initially, a cluster containing highmass stars formed in a molecular cloud. The winds and the ionizing radiation of the massive stars then created an expanding shell around this cluster. Gravitational instabilities in the expanding ring lead to fragmentation, cloud collapse and the formation of a second generation of stars, which now constitute the Cep OB2 association, which has a presumed age of about 5-7 Myr. The massive stars in Cep OB2 now affect the dense gas remaining from the parent shell and seem to trigger the formation of the third generation of stars in the dense cloud cores, including S140, along the edge of the bubble (see also Ábráhám et al. 2000).

The shape of the S140 cloud strongly suggests that the gas is being compressed by external pressure from the south-west direction. One source for this compression is the B0 star HD 211880, the exciting source of the S140 HII region, at a projected distance of $2.5 \mathrm{pc}$ from S140 IRS. Another factor may be the general expansion of the Cepheus bubble, the center of which is located to the south-west of the S140 cloud.

\subsection{Spatial distribution of the young stellar objects}

It is interesting to consider the spatial distribution of the cluster members. In Fig. 4 we plot the number of objects with $J-K^{\prime}>1.5$, i.e. objects that either show strong infrared excess or suffer strong extinction and therefore can be considered cluster member candidates, in several bins of radial separation from the center. One can see that the radial distribution of these stars is more or less uniform. The interpretation of this uniform radial distribution is not fully conclusive. We could interpret the embedded stars as objects which have formed at a uniform rate within a very dense central core over the star formation

\footnotetext{
${ }^{1}$ See e.g. Elmegreen \& Lada (1977) for an overview of triggered star formation in $\mathrm{OB}$ association, and e.g. Fukuda \& Hanawa (2000) for models of sequential triggered star formation by expansion of an HII region.
}

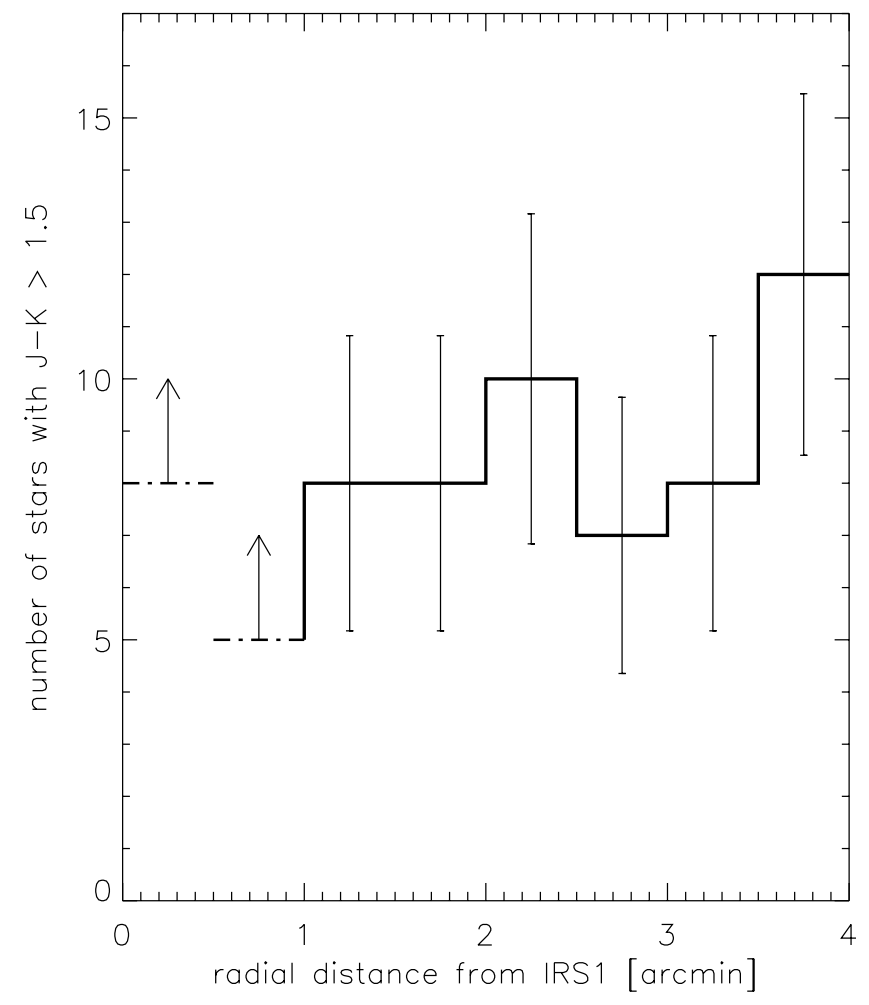

Fig. 4. Radial distance distribution of the stars around IRS 1. All objects with $J-K^{\prime}>1.5$ (corresponding to objects with strong infrared excess or suffering from at least $\sim 3$ mag of visual extinction) are considered to be cluster members. In the two innermost bins our sample is incomplete due to the very strong diffuse nebulosity, hampering the detection of faint point sources.

lifetime of S140 and which form an expanding spherical distribution. Or, the stars formed in a rapid burst with a linear distribution of escape speeds. Such a burst, followed by dispersal of a large fraction of dense ambient material (reducing the confining gravitational force), would result in the free expansion of the young stars. A third possibility is that the stars formed in situ with an efficiency related to both the local density and the compression of a passing shock wave which would sweep up material into an inner clumpy ring, possibly as now observed in CS with a radius of 30" (Hayashi \& Murata 1992). In this case, the stars could be younger. The age of the embedded stars could be tested by searching for excess $H-K$ band emission (the $H$-band filter failed during the run described here).

\section{Summary}

The main results of our study are as follows:

We find strong $\mathrm{H}_{2}$ shock emission around the $\sim 10 M_{\odot}$ protostar S140 IRS 1. Some of these shocks are related to the well known $\sim 160^{\circ} / 340^{\circ} \mathrm{CO}$ outflow, but the strongest shocks are found in the $20^{\circ} / 200^{\circ}$ outflow, which seems to be currently very active. These results provide evidence for the existence of two distinct bipolar outflow systems originating simultaneously from IRS 1 . The $\sim 8-10 M_{\odot}$ 
protostars IRS 1-3 are associated with a cluster of fainter sources, presumably lower-mass young stellar objects. The total mass in stars seems to exceed the mass of the remnant gas and dust in the S140 region significantly. This might indicate that the strong outflows of the protostars have expelled most of the gas from the region.

Acknowledgements. We would like to thank the Calar Alto staff for their support during our observations and Thomas Stanke for assistance in the data reduction. MDS is grateful to G. Weigelt for support and discussions during visits to the MPIfR.

\section{References}

Abraham, P., Balazs, L. G., \& Kun, M. 2000, A\&A, 354, 645

Armand, C., Baluteau, J.-P., Joubert, M., \& Cox, P. 1996, A\&A, 306, 593

Ashby, M. L. N., Bergin, E. A., Plume, R., et al. 2001, ApJ, 539, L119

Bally, J., \& Lada, C. J. 1983, ApJ, 265, 824

Bate, M. R., Bonnell, I. A., Clarke, C. J., et al. 2000, MNRAS, 317,773

Beichman, C. A., Becklin, E. E., \& Wynn-Williams, C. G. 1979, ApJ, 232, L47

Bernasconi, P. A., \& Maeder, A. 1996, A\&A, 307, 829

Bizenberger, P., McCaughrean, M. J., Birk, C., Thompson, D., \& Storz, C. 1998, Proc. SPIE, 3354, 825

Blair, G. N., Evans, N. J. II, Vandenbout, P. A., \& Petters, W. L. III 1978, ApJ, 219, 896

Brooke, T. Y., Sellgren, K., \& Smith, R. G. 1996, ApJ, 459, 209

Crampton, D., \& Fisher, W. A. 1974, Pub. Dom. Ap. Obs., 14, 283

Churchwell, E. 1997, ApJ, 479, L59

Eislöffel, J., Mundt, R., Ray, T. P., \& Rodriguez, L. F. 2000, in Protostars and Planets IV, ed. V. Mannings, A. P. Boss, \& S. S. Russel (Tucson: University of Arizona Press), 815

Elmegreen, B. G., \& Lada, C. J. 1977, ApJ, 214, 725

Fischer, O., Henning, Th., \& Yorke, H. W. 1996, A\&A, 308, 863

Fukuda, N., \& Hanawa, T. 2000, ApJ, 533, 911

Garay, G., \& Lizano, S. 1999, PASP, 111, 1049

Harker, D., Bregman, J., Tielens, A. G. G. M., Temi, P., \& Rank, D. 1997, A\&A, 324, 629

Harvey, P. M., Campbell, M. F., \& Hoffmann, W. F. 1978, ApJ, 219, 891

Hayashi, M., \& Murata, Y. 1992, PASJ, 44, 391

Hoare, M. G., Glindemann, A., \& Richichi, A. 1996, in The Role of Dust in the Formation of Stars, ed. H. U. Käufl, \& R. Siebenmorgen (Berlin: Springer), 35
Hoare, M. G., \& Muxlow, T. B. 1996, in Radio Emission from the Stars and the Sun, ed. A. R. Taylor, \& J. M. Paredes (San Francisco, ASP), ASP Conf. Ser., 93, 47

Kroupa, P. 2001, MNRAS, 322, 231

Kun, M., Balazs, L. G., \& Toth, I. 1987, ApSS, 134, 211

Labeyrie, C. D. 1970, A\&A, 6, 85

Lenzen, R. 1987, A\&A, 173, 124

Lester, D. F., Harvey, P. M., Joy, M., \& Ellis, H. B. Jr. 1986, ApJ, 309, 80

Mac Low, M.-M. 1999, ApJ, 524, 169

McCaughrean, M. J., Zinnecker, H., \& Rayner, J. T. 1994, ApJ, 436, L189

Minchin, N. R., White, G. J., \& Padman, R. 1993, A\&A, 277, 595

Minchin, N. R., Ward-Thompson, D., \& White, G. J. 1995, A\&A, 298, 894

Palla, F., \& Stahler, S. W. 2000, ApJ, 553, 299

Papaloizou, J. C. B., \& Terquem, C. 1995, MNRAS, 274, 987

Patel, N. A., Goldsmith, P. F., Heyer, M. H., Snell, R. L., \& Pratap, P. 1998, ApJ, 507, 241

Preibisch, Th., Balega, Y. Y., Schertl, D., Smith, M. D., \& Weigelt, G. 2001, A\&A, 378, 539

Rouan, D., Lena, P. J., Puget, J. L., de Boer, K. S., \& Wijnbergen, J. J. 1977, ApJ, 206, L35

Scalo, J. 1998, in The Stellar Initial Mass Function, ed. G. Gilmore, \& D. Howell, ASP Conf. Ser., 142, 201

Schertl, D., Balega, Y., Hannemann, T., et al. 2000, A\&A, 361, L29

Schwartz, P. R. 1989, ApJ, 338, L25

Smith, M. D. 1993, ApJ, 406, 520

Tanaka, M., Hasegawa, T., Hayashi, S. S., Brand, P. W. J. L., \& Gatley, I. 1989, ApJ, 336, 207

Terquem, C. 1998, ApJ, 509, 819

Tofani, G., Felli, M., Taylor, G. B., \& Hunter, T. R. 1995, A\&AS, 112, 299

van der Tak, F. F. S., Dishoeck, E. F., Evans II, N. J., \& Blake, G. A. 2000, ApJ, 537, 283

Weigelt, G. 1977, Opt. Commun., 21, 55

Weigelt, G., Preibisch, Th., Schertl, D., Balega, Y. Y., \& Smith, M. D. 2002, A\&A, 381, 905

Whitney, B. A., Kenyon, S. J., \& Gomez, M. 1997, ApJ, 485, 703

Wilner, D. J., \& Welch, W. J. 1994, ApJ, 427, 898

Yao, Y., Ishi, M., Nagata, T., et al. 1998, ApJ, 500, 320

Yu, K. C., Billawala, Y., Smith, M. D., Bally, J., \& Butner, H. M. 2000 AJ, 120, 1974

de Zeeuw, P. T., Hoogerwerf, R., de Bruijne, J. H. J., Brown, A. G. A., \& Blaauw, A. 1999, AJ, 117, 354

Zhou, S., Butner, H. M., Evans, N. J. II, et al. 1994, ApJ, 428, 219 